\title{
Coordinate flares observed by TRACE
}

\author{
Defang Kong, Xiaoli Yan and Zhike Xue \\ National Astronomical Observatories of China/Yunnan Astronomical Observatory, \\ Chinese Academy of Sciences, Kunming, Yunnan 650011, China \\ email: kdf@ynao.ac.cn
}

\begin{abstract}
Two flares occurred simultaneously in active region NOAA 9433 on 2001 April 25. The GOES X-ray fluxes show only one peak during the two flares. The negative magnetic polarities in the two flaring regions exhibited a clockwise rotation motion around the positive polarities, which can be seen from the evolution of the SOHO/MDI magnetograms. Through analyzing the potential fields extrapolated from the MDI magnetogram, we find that there is a channel strode by a group of magnetic field lines connecting the two flaring regions.
\end{abstract}

Keywords. Sun: activity, Sun: sunspots, Sun: flares

Sympathetic flares are defined as solar flares in different active regions that apparently occur as a common result of activation of a physical connection between the regions during a short period of time. Pearce \& Harrison (1990) analyzed the flare activity of 15 active region pairs and found that those active region pairs displayed a significant amount of coincident flaring. They suggested that sympathetic activity is triggered by the passage of some disturbance through magnetic connections. By analyzing the data of the magnetic fields, Bumba \& Klvana (1993) suggested that sympathetic flares may be related with the large-scale convective motion. Gopalswamy et al. (1999) also found that the X-ray ejecta from the eruption region triggered a sympathetic flare in an adjoining active region. Recently, Jiang et al. (2011) analyzed the coronal dimming formation after the first filament eruption which was associated with a CME and found that the dimming in the first eruption removed the overlying magnetic field on the other two filaments in quiet-Sun regions. Both quiescent filaments consecutively underwent the second and the third eruption.

The observation of Transition Region and Coronal Explorer (TRACE) covered the whole process of this event from white-light to EUV wavelength. The data of TRACE white-light and Fe Ix/x $171 \AA$ images have a cadence of about $30 \mathrm{~s}$ to 1 minute and a pixel size of 0.5 " (Handy et al. (1999). Full-disk line-of-sight magnetograms are used to show the magnetic fields in the photosphere. The magnetograms were taken by the Michelson Doppler Imager (MDI) on board the Solar and Heliospheric Observatory (SOHO; Scherrer et al. (1995) with a 96 minute cadence and a spatial resolution of $2^{\prime \prime} /$ pixel. In addition, we also use the data of soft-X-ray flux observed by Geostationary Operational Environmental Satellite (GOES) to identify the occurrence of flare.

The left panel of Fig. 1 shows the GOES X-ray fluxes from 10:00UT to 16:00UT on April 25, 2001. The GOES fluxes began to increase at 13:41UT. Next, an M-class flare occurred in active region NOAA 9433. The brightening of the two regions marked by two white arrows in active region NOAA 9433 can be seen from the middle panel of Fig. 1. The two patches began to brighten simultaneously. During the flare, the filament in this region did not erupt by analyzing the $\mathrm{H} \alpha$ data observed by Yunnan Astronomical Observatory. From the evolution of the magnetograms, the negative magnetic polarities marked by two white arrows in the right panel of Fig. 1 exhibited a rotation motion around the positive polarities in the two flaring regions. Figure 2 shows the potential fields extrapolated from 

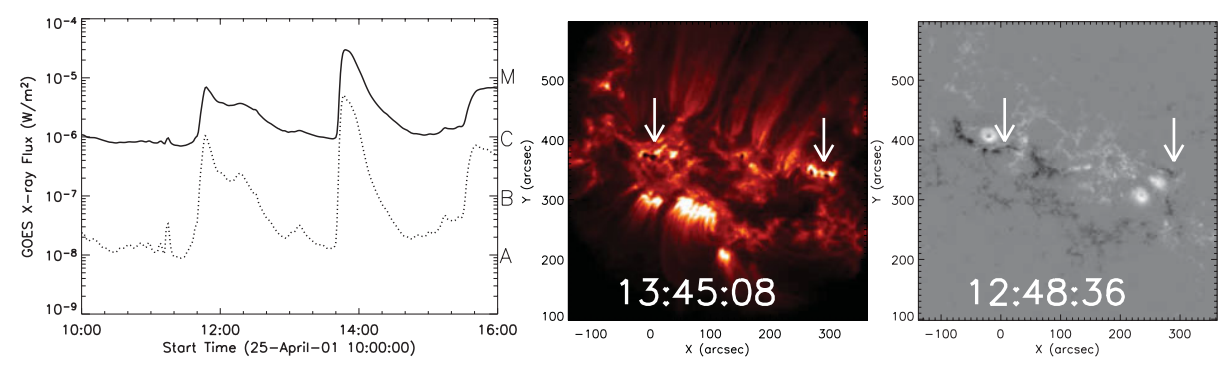

Figure 1. Left: Evolution of GOES soft-X-ray emission from 10:00UT to 16:00UT on April 25, 2001 (solid line: 1-8 $\AA$; dashed line: 0.5-4 $\AA$ ). Middle: $171 \AA$ image observed by TRACE; The white arrows indicate the two flaring regions. Right: SOHO/MDI magnetogram at 12:48:36UT; The negative magnetic polarities are marked by two white arrows.

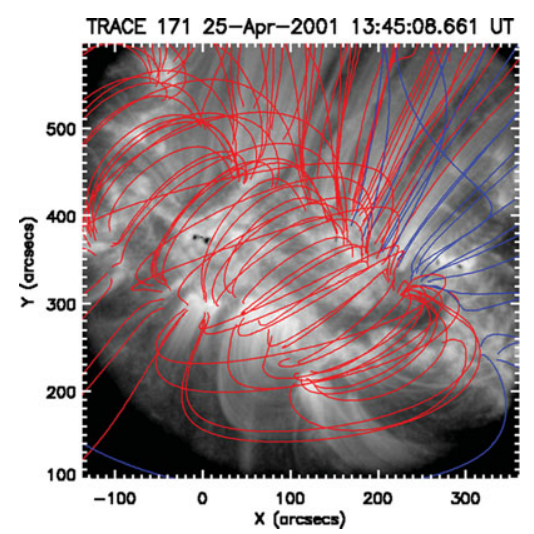

Figure 2. The potential fields extrapolated from the SOHO/MDI magnetogram at 12:00UT superimposed TRACE $171 \AA$ at 13:45UT on April 25, 2001.

the SOHO/MDI magnetograms at 12:00UT superimposed TRACE $171 \AA$ at 13:45UT on April 25, 2001. The red and blue lines indicate the closed and open magnetic field lines. From the potential fields lines, one can see that there is a channel strode by a group of magnetic field lines connecting the two flaring regions.

Two flares occurring simultaneously in one active region is very rare. One occurred near the leading sunspot and the other occurred near the following sunspot. The two flaring regions separated by $2 \times 10^{-5} \mathrm{~km}$. From the potential fields extrapolated from the MDI magnetogram, the two regions were connected by a channel strode by a group of magnetic field lines. The negative magnetic polarities near the positive magnetic polarities exhibited clockwise rotation motion before the occurrence of the flares. We assume that the two flaring regions must have a certain coupled magnetic fields and the sunspot rotation may play an important role in the occurrence of the flares (Yan et al. 2012a, $2012 \mathrm{~b})$. The mechanism deserved to be investigated further.

\section{References}

Pearce, G. \& Harrison, R. A. 1990, Astron. Astrophys., 228, 513

Bumba,V., Klvana, M. 1993, Astrophys. Space Sci., 199,45

Gopalswamy, N., Nitta, N., Manoharan, P. K., et al. 1999, Astron. Astrophys., 347, 684

Jiang, Y., Yang, J., et al. 2011, Astrophys. J., 738, 179

Handy, B. N., Acton, L. W., et al. 1999, Sol. Phys., 187, 229

Scherrer, P. H. et al. 1995, Sol. Phys., 162, 169

Yan, X. L., Qu, Z. Q., \& Kong, D. F., 2012, Astronomical J., 143, 56

Yan, X. L., Qu, Z. Q., Kong, D. F., \& Xu, C. L., 2012, Astrophys. J., 754, 16 